\title{
Successful digital asset management systems rely on making friends and influencing people
}

\author{
Duncan Daines \\ is Client Director and Knowledge Leader for digital branding at Landor, where he leads brand asset management programs for such \\ clients as BP, bmi, Belgacom and Alpharma. Previously, he was a brand consultant at Sapient and prior to that he was responsible for \\ the communication of the British Airways masterbrand and its sub-brands across all digital channels. Duncan regularly contributes to \\ the media, and more recently spoke at Henry Stewart's 7th Annual Digital Asset Management Symposium in London in 2004.
}

Keywords: people centered, project champions, stated requirements, actual requirements, tools, adoption

Abstract Technology can only do so much when it comes to digital asset management (DAM). Knowing your audiences, what they want and why they want it is even more important than what the technology is capable of providing. Without their cooperation even the most advanced and intelligent systems will prove to be a waste of money. This paper explores the major personality types present in most organizations and how to stack the odds in your favor to encourage a successful DAM program.

\section{INTRODUCTION}

Science and Technology revolutionize our lives, but memory, tradition and myth frame our response. Expelled from individual consciousness by the rush of change, history finds its revenge by stamping the collective unconscious with habits, values, expectations, dreams. ${ }^{1}$

The author has been delivering and implementing digital brand asset management systems since 1998 and has had the privilege of working with some of the world's most sophisticated and switched-on organizations, from BP to bmi and Ferrari. In the last six years the technology side of the equation has, without question, reached ever-greater levels of wizardry. Its development has been dynamic to say the least and it can now provide an impressive range of tools that can be tailored to create a vast range of solutions.

The other side of the equation, however, doesn't stack up in quite the same way.

Duncan Daines Landor Associates, Klamath House, 18 Clerkenwell Green London EC1R ODP

Tel: +44 (0)207880 8000 E-mail: DUNCAN_DAINES@ uk.landor.com

\section{TECHNOLOGY VERSUS PEOPLE}

People's job-specific needs may be different and their approach may vary wildly, but their fundamental needs, as human beings, remain constant. In other words, the human element of
DAM is much the same as it has always been. And it is all too easy to get fixated on what the technology can do rather than how much and what people - the end users - will get out of it.

The following are examples of real objectives set for real DAM projects.

- To consolidate, optimize, define and communicate global development and trafficking solutions (incorporating localized variations and expertise), providing transparency to processes and support structures across product type and locations.

- To implement a global budget planning and tracking solution, not only providing transparency to the time and money spent within this area, but also improving the ability to analyze future efficiencies.

These objectives were authored by some of the largest and most respected brand owners in the world. These are companies whose brands are worth billions. Operationally these objectives are absolutely sound - you cannot disagree with the probable returns on investment or the rationale behind them. The problem is that they bear no reference whatsoever to the human element; to how people react with it and what they will use DAM for. Neither the objectives 
nor the wider strategies in which they were framed reflect the need to recognize the people involved in the process and how they will benefit from it. And that is the crux of the problem. Technology can provide solutions, but if your people don't make use of them you can end up spending a great deal for a very modest return.

People are funny creatures. They need to feel ownership of some sort and to some extent they like to feel in control of their working practices. They need motivational triggers before they will adopt new systems and those triggers must be relevant and meaningful to them as individuals. People cherry-pick processes and methods of working to suit themselves in the belief that selfselection is the most appropriate and efficient approach. You can try to force them to change, but rarely will you succeed.

DAM is all about effective brand asset management. Intelligent, enlightened and perceptive people management is crucial to its success. The first step in any successful DAM project is to define your requirements and to do that you must know and understand your audience and its various motivational triggers.

\section{STEP 1: KNOW YOUR PEOPLE}

Every organization is made up of different sorts of characters who are at different life stages within that company. Broadly there are four types, according to a psychographic segmentation study conducted by Landor's sister company Research International. They are Players, Soloists, Drummers and Novices (see Figure 1).

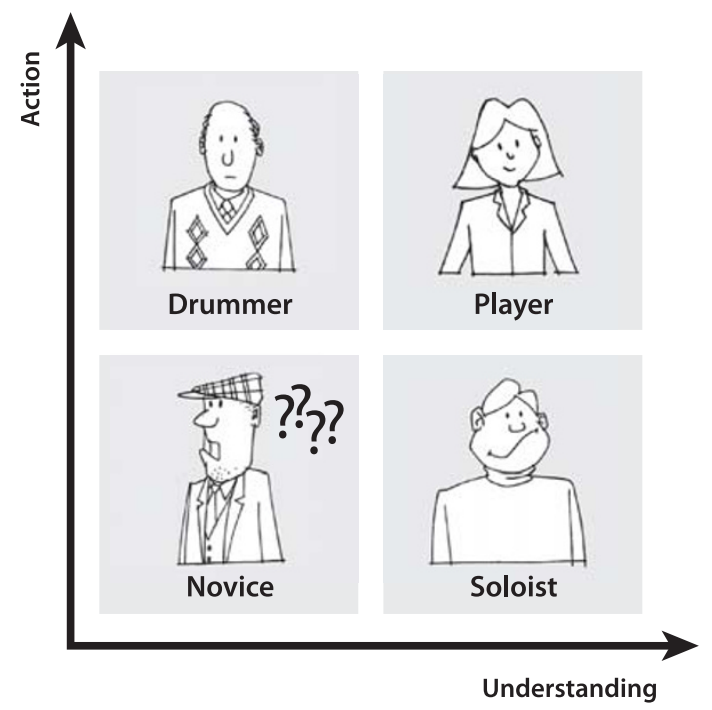

Figure 1: Action vs understanding Source: Research International

Novices are usually low-end employees. They are often new or new-ish to the company, frequently contract staff, have a very 'jobsworthy' attitude and a cynical outlook. They gravitate towards administrative roles, but there are a surprisingly high percentage of them in departments, such as marketing communications, human resources and research and development (see Figure 2). Typically they don't care about the company's brand values and are not sure about its goals; have poor leadership skills and organizational efficiency and low understanding of customer needs and what will make the business successful. Basically they just get on with their job and aren't greatly interested in much else.

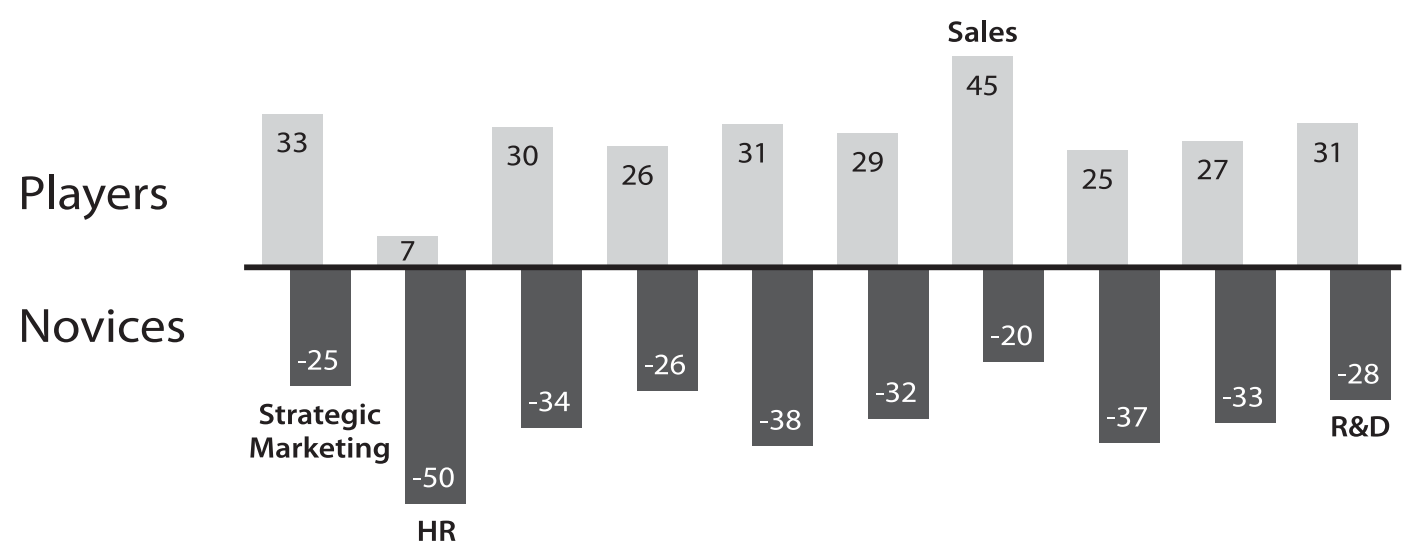

Figure 2: Key departments

Source: Research International 
Drummers share several tendencies with Novices, but manifest some important - and positive - differences. Like Novices, they lack understanding of customer needs and what will make the business successful, and tend to feel fairly disenfranchised by the company. They are committed to work, often staying in the same position for five years or more, but do not get satisfaction and are frustrated easily. They tend to be very process-oriented: they understand the process, but not the wider view of why it is being changed.

Soloists, as the appellation suggests, generally have their own agenda to pursue. Not surprisingly, they often work in silos. They are talented individuals - with the emphasis on individual - who make excellent practitioners, but poor leaders, particularly in senior management roles. Not that this necessarily prevents their ascent of the career ladder. They know what the brand stands for and have high understanding of customer needs and what will make the business successful. Usually, however, they are too busy concentrating on their virtuoso performance to commit to corporate goals.

Players are an asset to any organization. They know the brand and believe in it and they demonstrate excellent knowledge and understanding of the broader brand context. They are ambitious people who are not afraid to take risks, personally and professionally, and who are committed to furthering the corporate cause. They make superb senior managers as

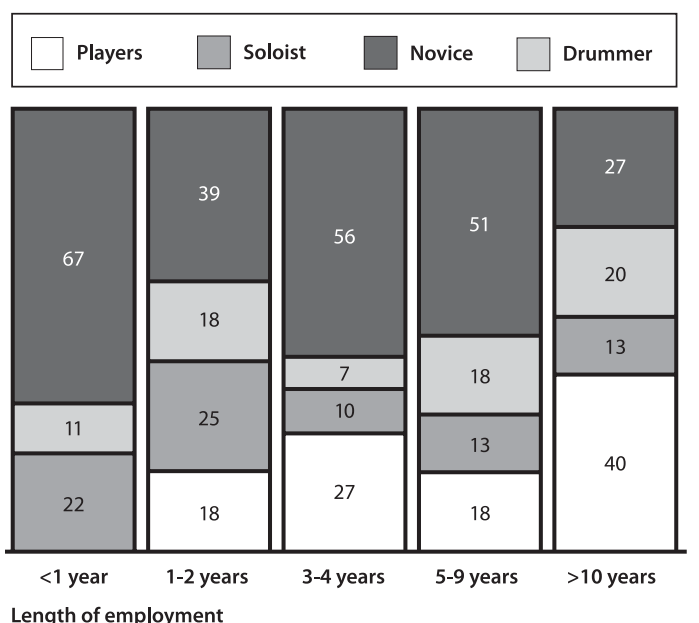

Length of employment

Figure 3: Employee lifestage

Source: Research International they are supporting and involving leaders and have high levels of customer and business competence. As you would expect, they tend to achieve high levels of creativity and speed and score highly on both productivity measures and customer satisfaction ratings. They are frequently found in sales departments and remain among the most loyal employees.

\section{STEP 2: WHAT'S THE MOTIVATION?}

Each of these groups has different motivational triggers and communications needs. When planning a DAM program you have to be ultra conscious of the balance of your audience and to plan accordingly. You have to pin down who people are, in which departments and at what lifestage within the organization (see Figure 3). Requirement definition is a crucial element in any successful DAM strategy. As well as determining what people need and how you are going to supply it, it is vital that you weight different groups' hierarchy of needs.

Concentrating too much on the needs of Novices who are likely to stay with the company for less than a year will put you on the road to failure before you have even begun to implement the project. Equally, you can't afford to have your requirements skewed too far towards people who can reel off the longest wish lists, but are among the most resistant to change ie Drummers. Neither can you focus too narrowly on Players at the expense of those who will be responsible for using the new system. It is about achieving the right balance.

No DAM system is a panacea. Change is disruptive and will meet with inherent resistance and it is therefore vital that you present it in the most appropriate way to its various audiences, utilize their different strengths and use other mechanisms and support structures to ease its passage. Supplying someone with a checklist of things the new system dictates isn't going to add anything to their day and is all but begging to be rejected. Presenting it in a way that is fun and challenging, and backing it up with an award or reward mechanism is a much more motivating proposition.

\section{STEP 3: PROJECT OWNERSHIP}

Each group's motivational triggers need to be addressed and each needs to be given an 
appropriate level of ownership of the project to ensure its success.

Players are characterized by a "can do, let's go" attitude and are probably any DAM initiative's most valuable allies. To get the most out of their support, do keep their motivation levels high; empower them to lead change with cross-functional tasks and allow them to champion the project. Give them the authority to make things happen. Don't let them become de-motivated, because they will run out of steam or leave, and don't provide them with complete solutions that they just implement.

Soloists can also be important allies if you provide them with the bigger picture. Do utilize their talents by integrating them into the team; focus them to align their activities with higherorder goals; provide team-based tasks and encourage and reward them as team players. Like Players they will not respond to complete solutions that deny them ownership of any sort and you need to guard against them becoming de-motivated. However, you must also prevent them from controlling the agenda, which they are wont to do.

Drummers require a different approach they want proof that something works and that it will help them to do their job better. They want tangible benefits and they want them now. Do find a context in which the initiative can be discussed in a meaningful way as far as they are concerned; provide them with "out of the box" tools; use Players to lead by example and inspire them; constantly prove that the new way works and is an improvement on the old, and push them to adapt. Don't use jargon; communicate the initiative in ways that they cannot action or allow them to undermine changes.

Novices loathe change; they rarely see the need for it and are only interested in new initiatives if they are communicated in a way that makes direct sense to them. For them everything is hassle. Like Drummers they need relevant context and inspiration and leadership from Players. They don't need jargon and mustn't be allowed to undermine changes. Do set targets that stretch them and require change, and educate them rather than just inform them. Don't expect them to get it after just seeing a presentation.

Players and Soloists tend to be influencers while Drummers and Novices generally act as underminers. That said, all could be constructive or destructive forces depending on how they are handled.

Maximize the project's support system by ensuring that likely influencers are on its steering group. Empower them to resolve operational blocks with Novices and Drummers; create key performance indicators that are written into their personal assessments and let them own the rollout of the project, from planning to execution.

Minimize the potential for underminers by providing them with explicit rewards for adoption and use and create suitable key performance indicators within their contracts. Address all their process-based requests within a phased scope of work and actively communicate the phasing of the roll-out. Continue to reiterate their importance and the importance of their involvement in the overall success of the venture. Allow Drummers to feed into the team on processes that they own or control.

Communicate the overarching importance of the DAM initiative at every level and be as directive as possible without being dictatorial. Leave your audiences in no doubt as to the benefits of its success and their roles within it. Bear in mind that DAM operates in a brand environment which is seen as fundamentally creative, and that creativity is a hugely motivating factor. Creativity without order is chaos, but too much order, or order that is too rigorously imposed squashes creativity. And remember that no system — no matter how sophisticated - will effect operational and behavioral change overnight.

\section{TOP TIPS FOR SUCCESS (OR FAILURE)}

The author has been involved in the implementation of many highly successful DAM systems and also one that was dropped after just 16 months. Failure is usually more instructive than success and it is fair to say that some vital lessons were learned through it. In that context I'd like to offer both a caveat emptor list and my tips for success.

A DAM initiative will not prosper if:

- the entire project is focused on technology and not on people;

- a perception exists that it will immediately make the brand more consistent or deliver immediate return on investment - it won't; 
- it is assumed that everyone will love it and use it religiously - they won't;

- employees have no comprehension as to what its role is or how to use it;

- it is perceived as a "big brother" initiative imposed on the masses by corporate headquarters or centralized management;

- it disenfranchises employees whose role is to work with agencies to produce creative work;

- agencies and other suppliers are not compelled to use it and there is a lack of agency adoption or responsibility to use the tools;

- it is a project not a long-term commitment;

- nothing is measured;

- it is seen as a panacea for poor assets.

It will be a success if:

- the project is people-centered not technologyoriented;

- the project is seen as imperative to the achievement of greater goals rather than just another "project";

- project champions are developed and fostered at every level and at every phase of the project;

- the organization's requirements are well defined and understood in the context of the various audiences that make it up;
- stated requirements are actual requirements;

- the tools offered reflect the needs of your audiences, not your needs;

- the tools are presented as a benefit to people rather than a means of delivering return on investment;

- you are as directive as your corporate culture allows;

- key early adopters are chosen carefully;

- you stack support in your favor;

- performance, reward and support structures are put in place;

- people are held to be accountable for its adoption and use;

- training in context and on the job is thoughtfully applied;

- introductions are phased so that initial issues or difficulties can be worked out in advance of a company-wide roll out;

- it is kept top of mind by keeping it in the news;

- you nurture your champions and the businesses they represent for the long term;

- you start small and evolve, developing success stories as you go.

\section{Reference}

1 Schlesinger Jr, A. M. (1986) "The Challenge of Change", New York Times Magazine, July 27. 